\title{
Complexity reduction in low-delay Farrow- structure-based variable fractional delay FIR filters utilizing linear-phase subfilters
}

Amir Eghbali and Håkan Johansson

Linköping University Post Print

N.B.: When citing this work, cite the original article.

(C)2012 IEEE. Personal use of this material is permitted. However, permission to reprint/republish this material for advertising or promotional purposes or for creating new collective works for resale or redistribution to servers or lists, or to reuse any copyrighted component of this work in other works must be obtained from the IEEE.

Amir Eghbali and Håkan Johansson, Complexity reduction in low-delay Farrow-structurebased variable fractional delay FIR filters utilizing linear-phase subfilters, 2012, Eur. Conf. Circuit Theory Design, IEEE.

http://dx.doi.org/10.1109/ECCTD.2011.6043300

Postprint available at: Linköping University Electronic Press http://urn.kb.se/resolve?urn=urn:nbn:se:liu:diva-84866 


\title{
Complexity Reduction in Low-Delay Farrow-Structure-Based Variable Fractional Delay FIR Filters Utilizing Linear-Phase Subfilters
}

\author{
Amir Eghbali and Håkan Johansson \\ Division of Electronics Systems, Department of Electrical Engineering, \\ Linköping University, Sweden. E-mail: \{amire,hakanj\}@isy.liu.se.
}

\begin{abstract}
This paper proposes a method to design low-delay fractional delay (FD) filters, using the Farrow structure. The proposed method employs both linear-phase and nonlinearphase finite-length impulse response (FIR) subfilters. This is in contrast to conventional methods that utilize only nonlinear-phase FIR subfilters. Two design cases are considered. The first case uses nonlinear-phase FIR filters in all branches of the Farrow structure. The second case uses linear-phase FIR filters in every second branch. These branches have milder restrictions on the approximation error. Therefore, even with a reduced order, for these linear-phase FIR filters, the approximation error is not affected. However, the arithmetic complexity, in terms of the number of distinct multiplications, is reduced by an average of $30 \%$. Design examples illustrate the method.
\end{abstract}

Index Terms-Farrow Structure, Low-Delay, Fractional Delay, Low-Complexity.

\section{INTRODUCTION}

Applications such as medical signal processing [1], digital communications [2], time/delay estimation [3], and nonuniform sampling [4] require either to perform sampling rate conversion (SRC) or to delay a digital signal by fractions of the sampling period. A software defined radio may require both of these [5]. For example, one may perform SRC and also estimate the phase/carrier. In multistandard receivers, there is a need to dynamically perform SRC and signal delaying so as to support various standards. We can use dedicated blocks to perform SRC and signal delaying, for each standard, but this requires to either (i) design a large set of filters offline, or (ii) design the filters online.

An efficient way, to solve the above problem, is to use the Farrow structure [6]. The Farrow structure is designed to approximate an allpass function with an adjustable fractional delay (FD) of $\mu(n)$. The FD value can change during each sampling period. For SRC, one should delay each input sample by a specific $\mu(n)$ whereas for signal delaying, all input samples are delayed by a fixed $\mu(n)$. For simplicity, the rest of the paper uses the term $\mu$ instead of $\mu(n)$.

\section{A. Contribution of the Paper}

The Farrow structure, shown in Fig. 1, is generally composed of $L+1$ fixed finite-length impulse response (FIR) subfilters ${ }^{1} S_{k}(z)$ and variable multipliers $\mu$. By a proper design of $S_{k}(z)$, the Farrow structure can approximate FD filters with an adjustable $\mu$. With realizable (nonideal) filters, the resulting FD filters will have an approximation error which can be reduced as in, e.g., [7]-[12].

The majority of the earlier design methods choose $S_{k}(z)$ to be linear-phase FIR filters. Although this reduces the arithmetic complexity, because of the resulting symmetry or antisymmetry in the impulse responses of $S_{k}(z)$, the overall delay increases. This is partly due to the strict constraints which are inherently imposed on some of $S_{k}(z)$ thereby increasing the values of $N_{k}$. With odd $N_{k}$, the subfilters in branches $k=0,2,4, \ldots$, have a higher order as compared to other branches [8]. For even $N_{k}$, the subfilter $S_{0}(z)$ is a pure delay and, then, the subfilters in branches $k=1,3, \ldots$, have a higher order [8].

This paper proposes a method to design FD filters, with odd $N_{k}$, in which the Farrow structure uses both nonlinear-phase and linear-phase FIR subfilters. In other words, we replace the high-order linear-phase FIR subfilters $S_{k}(z), k=0,2, \ldots$, with lower-order nonlinear-phase FIR filters. By means of design examples, we show that this reduces the arithmetic complexity, as opposed to the case with nonlinear-phase FIR filters in all branches.

This paper mainly considers cases where the nonlinearphase subfilters have the same orders in all branches. Also, the linear-phase subfilters have the same orders. The design examples show that we can indeed meet the specifications with a reduced arithmetic complexity. However, the paper also outlines some design examples to show that we can allow subfilters of different orders, in different branches, thereby further reducing the arithmetic complexity. This has not been treated earlier for low-delay FD filters. We consider minimax designs by minimizing the maximum of the modulus of the complex error, i.e., the difference between the frequency responses of (i) the nonideal Farrow structure, and (ii) the ideal FD filter. Note that the proposed methods can also be applied if $N_{k}$ is even.

\footnotetext{
${ }^{1}$ The subfilters can alternatively be infinite-length impulse response (IIR) but this paper focuses on the FIR case.
} 


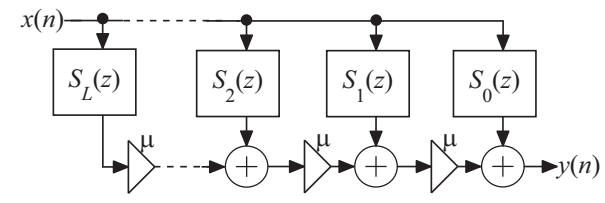

Fig. 1. Farrow structure with fixed subfilters $S_{k}(z)$ and variable FD $\mu$.

TABLE I

POSSIBLE TYPES OF LINEAR-PHASE FIR FILTER $S_{k}(z)$.

\begin{tabular}{|c|c|c|}
\hline$N_{k}$ & $k$ & Type \\
\hline even & even & I \\
\hline even & odd & III \\
\hline odd & even & II \\
\hline odd & odd & IV \\
\hline
\end{tabular}

\section{B. Paper Outline}

Section II outlines the Farrow structure and the error frequency responses. In Section III, the filter design problem is treated and a discussion on the arithmetic complexity is provided. Further, some design examples are also illustrated. Finally, Section IV concludes the paper.

\section{FARrow Structure}

The Farrow structure is composed of fixed general FIR subfilters $S_{k}(z), k=0,1, \ldots, L$, of order $N_{k}$ where the transfer function is [6]

$$
H(z, \mu)=\sum_{k=0}^{L} S_{k}(z) \mu^{k}, \quad|\mu| \leq 0.5 .
$$

Here, $\mu$ is the FD value. If $S_{k}(z)$ are chosen to exhibit a linear phase, they could have any of the four types [13] of linear-phase FIR filters as in Table I.

\section{A. Error Frequency Responses}

Generally, $S_{k}(z), \quad k=0,1, \ldots, L$, are designed so as to approximate the following desired causal complex and unwrapped phase responses

$$
\begin{aligned}
H_{\mathrm{des}}\left(e^{j \omega T}, \mu\right) & =e^{-j(\Delta+\mu) \omega T}, \\
\Phi_{\mathrm{des}}(\omega T, \mu) & =-(\Delta+\mu) \omega T
\end{aligned}
$$

where $\omega T \in\left[0, \omega_{c} T\right]$. If all $S_{k}(z)$ are linear-phase FIR filters, the overall delay is defined as

$$
\Delta=\frac{\max _{k}\left(N_{k}\right)}{2} \text {. }
$$

With linear-phase FIR subfilters, the value of $\Delta$ can be intolerably large due to the strict constraints on some of $S_{k}(z)$ [8]. If

$$
H\left(e^{j \omega T}, \mu\right)=\left|H\left(e^{j \omega T}, \mu\right)\right| e^{j \Phi(\omega T, \mu)},
$$

the complex, magnitude, and phase errors are

$$
\begin{aligned}
H_{c}^{e}\left(e^{j \omega T}, \mu\right) & =H\left(e^{j \omega T}, \mu\right)-e^{-j(\Delta+\mu) \omega T}, \\
H_{m}^{e}(\omega T, \mu) & =\left|H\left(e^{j \omega T}, \mu\right)\right|-1, \\
H_{p}^{e}(\omega T, \mu) & =\Phi(\omega T, \mu)+(\Delta+\mu) \omega T .
\end{aligned}
$$

With a known bound on $\left|H_{c}^{e}\left(e^{j \omega T}, \mu\right)\right|$, the bounds on $H_{m}^{e}(\omega T, \mu)$ and $H_{p}^{e}(\omega T, \mu)$ can be obtained [8].

\section{Filter Design}

Some applications may require to have $\Delta<\frac{\max _{k}\left(N_{k}\right)}{2}$ which would further restrict $S_{k}(z)$ thereby increasing $N_{k}$. With odd $N_{k}$, this restriction mainly increases the orders of $S_{k}(z), k=0,2, \ldots$. To alleviate this, one can increase the degrees of freedom, in the filter design, by using general nonlinear-phase FIR filters for all $S_{k}(z)$. Although this gives low-delay FD filters, it unnecessarily increases the arithmetic complexity.

This paper shows that the arithmetic complexity can be reduced by allowing some of $S_{k}(z)$ to be linear-phase and keeping the others as nonlinear-phase. With odd $N_{k}$, this amounts to having nonlinear-phase FIR filters in branches $k=$ $0,2, \ldots$, and linear-phase FIR filters in branches $k=1,3, \ldots$. In the examples of this paper and for a low-delay specification, we use a new $\Delta$ which is around $60 \%$ of that given by (4). For the same approximation error, this new $\Delta$ can further be reduced by increasing $N_{k}$.

With a given $\Delta$ and $\omega_{c} T$, we design two FD filters for comparison purposes. In the first one, all $S_{k}(z)$ are nonlinearphase FIR filters having the same orders. For the second FD filter, we reduce the orders of $S_{k}(z), k=1,3, \ldots$, and also replace them with Type IV linear-phase FIR filters. The filter design problem, considered here, is formulated as

$$
\min \delta \text { subject to }
$$

$$
\left|H_{c}^{e}(\omega T, \mu)\right| \leq \delta
$$

where $\omega T \in\left[0, \omega_{c} T\right]$. This design problem is convex and it can be solved using the standard filter design methods employing, for example, the real rotation theorem. In this paper, we use the algorithm in fminimax to solve (9). In the examples of Section III-B, we have the same orders $N_{k}$ in branches $k=0,2, \ldots$. Similarly, the subfilters in branches $k=1,3, \ldots$, have the same orders. However, as we shall show in Section III-C, it is possible to obtain low-delay FD filters in which $S_{k}(z)$ have different orders in different branches. This can further reduce the arithmetic complexity.

\section{A. Arithmetic Complexity}

For comparison, we use the arithmetic complexity in terms of the number of distinct multiplications, as multipliers are more costly to implement than adders. If all $S_{k}(z)$ are general nonlinear-phase FIR filters of orders $N_{k}$, the multiplicative complexity of the Farrow structure is

$$
C_{g}=\sum_{k=0}^{L}\left(N_{k}+1\right)
$$

By replacing some of $S_{k}(z)$ with Type IV linear-phase FIR filters, the multiplicative complexity becomes

$$
C_{l}= \begin{cases}\sum_{k=0}^{\frac{L-1}{2}}\left(N_{2 k}+1\right)+\sum_{k=0}^{\frac{L-1}{2}} \frac{N_{2 k+1}+1}{2} & \text { odd } L \\ \sum_{k=0}^{\frac{L}{2}}\left(N_{2 k}+1\right)+\sum_{k=0}^{\frac{L}{2}-1} \frac{N_{2 k+1}+1}{2} & \text { even } L .\end{cases}
$$


(a) $\mathrm{N}_{\mathrm{k}}=\left[\begin{array}{lllll}11 & 11 & 11 & 11 & 11\end{array}\right]$

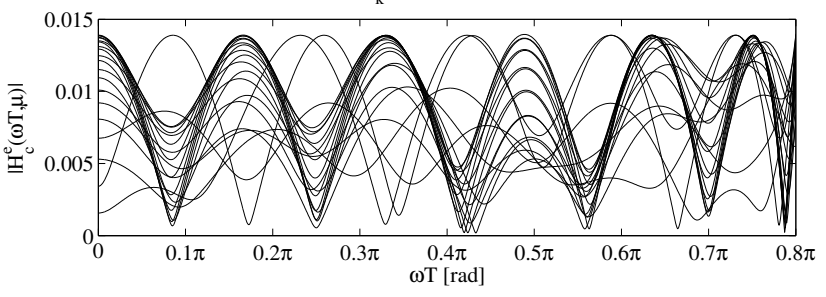

(b) $\mathrm{N}_{\mathrm{k}}=\left[\begin{array}{lllll}11 & 7 & 11 & 7 & 11\end{array}\right]$

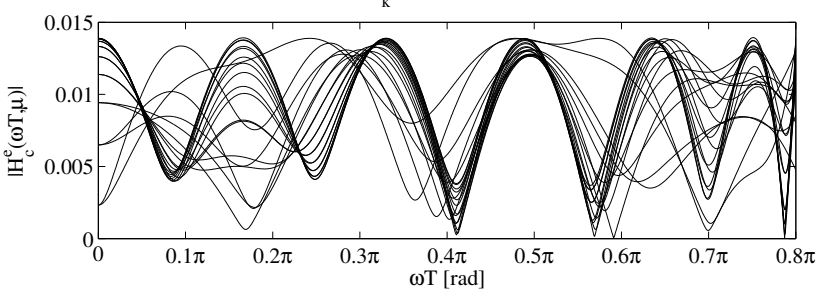

Fig. 2. Complex error for low-delay FD filters with $\Delta=3.5$.

In other words, complexity savings occur in the branches utilizing linear-phase FIR $S_{k}(z)$. This saving is due to (i) a smaller $N_{k}$, and (ii) the inherent antisymmetry in the impulse responses of Type IV linear-phase FIR filters. Note that the proposed method reduces the number of adders as well, but to a lower extent.

\section{B. Design Examples}

This section outlines some design examples and the corresponding savings in the multiplicative complexity. In all of these examples, the two FD filters meet practically the same specifications.

Example 1: If $\omega_{c} T=0.8 \pi, \Delta=3.5$, and $L=4$, the case with nonlinear-phase $S_{k}(z)$ and $N_{k}=\{11,11,11,11,11\}$ gives $\delta \approx 0.014$. With nonlinear-phase $S_{k}(z), k=0,2, \ldots$, and linear-phase $S_{k}(z), \quad k=1,3, \ldots$, where $N_{k}=$ $\{11,7,11,7,11\}$, the multiplicative complexity is reduced by $27 \%$. The characteristics of these FD filters are shown in Fig. 2.

Example 2: With $\omega_{c} T=0.5 \pi, \Delta=4.5$, and $L=3$, nonlinear-phase $S_{k}(z)$ with $N_{k}=\{13,13,13,13\}$ gives $\delta \approx 0.002$. If $S_{k}(z), k=0,2, \ldots$, are nonlinear-phase and $S_{k}(z), k=1,3, \ldots$, are linear-phase where $N_{k}=$ $\{13,9,13,9\}$, the multiplicative complexity is $32 \%$ lower resulting in the filter charactersitics of Fig. 3.

Example 3: For $\omega_{c} T=0.7 \pi, \Delta=2.5, L=4$, and nonlinear-phase $S_{k}(z)$ with $N_{k}=\{9,9,9,9,9\}$, we have $\delta \approx 0.006$. The choice of nonlinear-phase $S_{k}(z), k=$ $0,2, \ldots$, and linear-phase $S_{k}(z), k=1,3, \ldots$, where $N_{k}=$ $\{9,5,9,5,9\}$, gives the filter characteristics of Fig. 4 in which the multiplicative complexity is $28 \%$ lower.

Example 4: Setting $\omega_{c} T=0.9 \pi, \Delta=5.5$, $L=5$, and choosing nonlinear-phase $S_{k}(z)$ with $N_{k}=$ $\{17,17,17,17,17,17\}$ results in $\delta \approx 0.035$. The choice of nonlinear-phase $S_{k}(z), \quad k=0,2, \ldots$, and linear-phase $S_{k}(z), \quad k=1,3, \ldots$, where $N_{k}=\{17,11,17,11,17,11\}$,
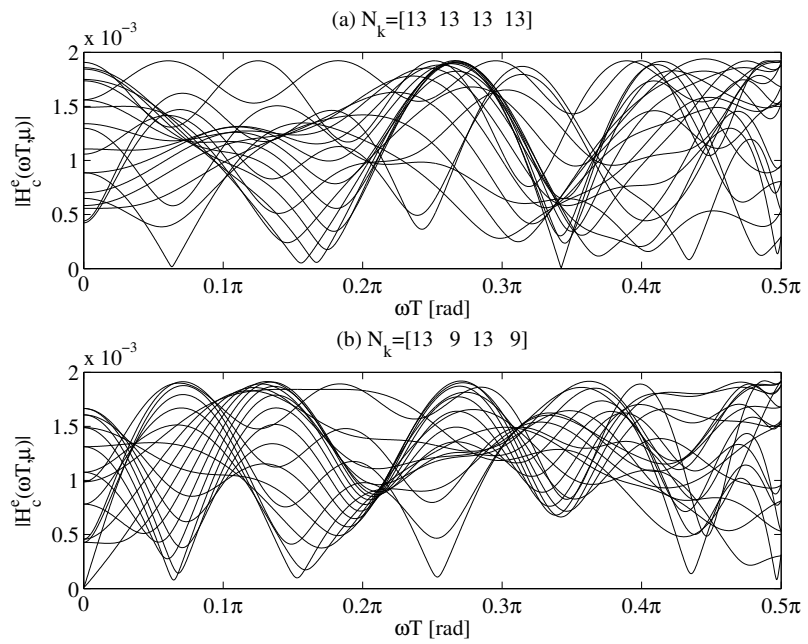

Fig. 3. Complex error for low-delay FD filters with $\Delta=4.5$.
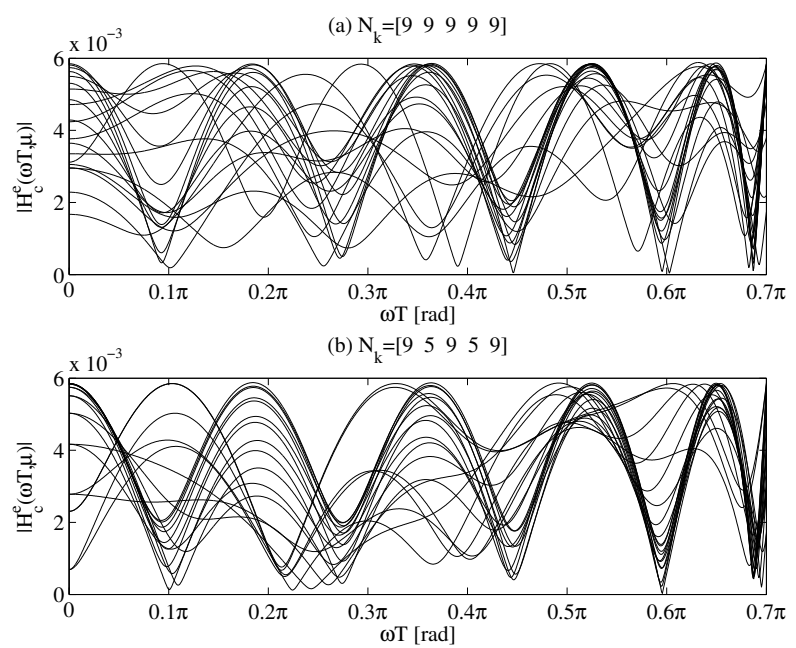

Fig. 4. Complex error for low-delay FD filters with $\Delta=2.5$.

gives a multiplicative complexity which is $33 \%$ lower. The filter characteristics are shown in Fig. 5.

Example 5: If $\omega_{c} T=0.6 \pi, \Delta=4.5, L=3$, and with nonlinear-phase $S_{k}(z)$ in which $N_{k}=\{15,15,15,15\}$, we get $\delta \approx 0.004$. With nonlinear-phase $S_{k}(z), k=0,2, \ldots$, and linear-phase $S_{k}(z), k=1,3, \ldots$, where $N_{k}=\{15,9,15,9\}$, we have a $35 \%$ lower multiplicative complexity. The filter characteristics are shown in Fig. 6.

\section{Subfilters with Different Orders}

As $k$ increases, the constraints on $S_{k}(z)$ become milder thereby allowing us to reduce the value of $N_{k}$ [8]. Therefore, one can solve (9) in a general form with different values of $N_{k}$ in different branches where some of the nonlinear-phase subfilters can also be replaced with linear-phase subfilters. Hence, the arithmetic complexity can further be reduced. This section provides examples to illustrate this.

Example 6: With the specifications in Example 4, i.e., $\omega_{c} T=0.9 \pi, \Delta=5.5$, and $L=5$, we can use subfil- 
(a) $\mathrm{N}_{\mathrm{k}}=\left[\begin{array}{llllll}17 & 17 & 17 & 17 & 17 & 17\end{array}\right]$

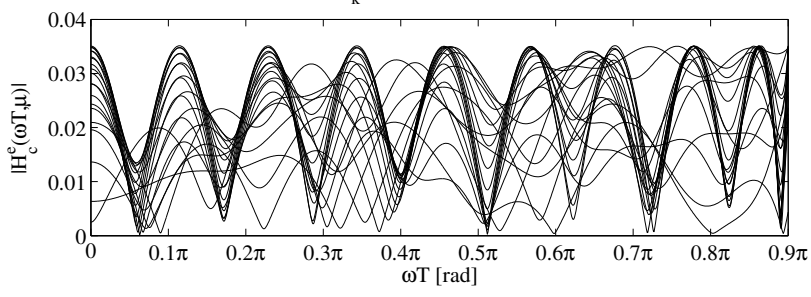

(b) $\mathrm{N}_{\mathrm{k}}=\left[\begin{array}{llllll}17 & 11 & 17 & 11 & 17 & 11\end{array}\right]$

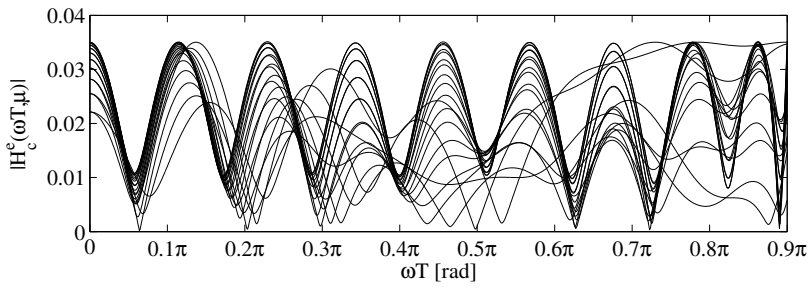

Fig. 5. Complex error for low-delay FD filters with $\Delta=5.5$.

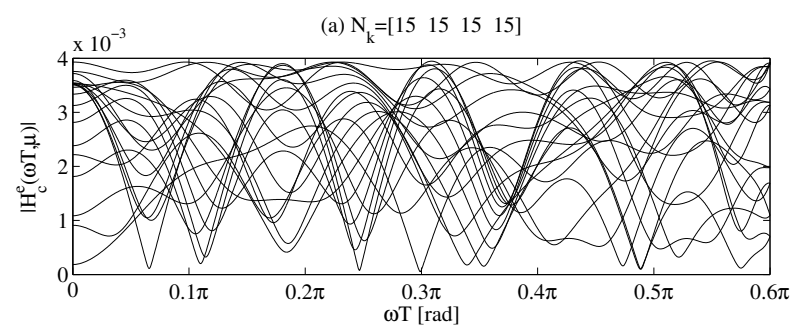

(b) $\mathrm{N}_{\mathrm{k}}=\left[\begin{array}{llll}15 & 9 & 15 & 9\end{array}\right]$

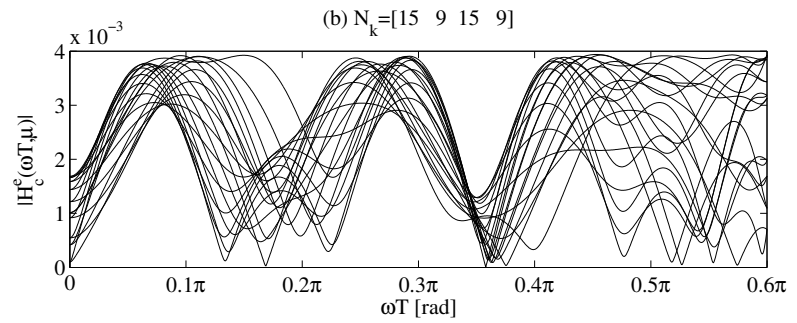

Fig. 6. Complex error for low-delay FD filters with $\Delta=4.5$.

ters of different orders. Here, nonlinear-phase $S_{k}(z), k=$ $0,2, \ldots$, and linear-phase $S_{k}(z), k=1,3, \ldots$ where $N_{k}=$ $\{17,11,13,11,5,3\}$ gives the same approximation error. The multiplicative complexity is reduced by an additional $27 \%$, as opposed to that of Fig. 5(b). The characteristics of these FD filters are shown in Fig. 7(a).

Example 7: Here, we use the specifications in the previous example but $S_{4}(z)$ has an order of $N_{4}=5$ and it is also a Type IV linear-phase FIR filter. This shows that some of the nonlinear-phase $S_{k}(z)$ can indeed be replaced with linearphase FIR filters thereby further reducing the multiplicative complexity. The filter characteristics are shown in Fig. 7(b).

\section{CONCLUSION}

A method for designing low-delay FD filters was outlined in which both linear-phase and nonlinear-phase subfilters are used. As opposed to the case with nonlinear-phase FIR filters, in all branches of the Farrow structure, the proposed method uses linear-phase FIR filters in every second branch. Even

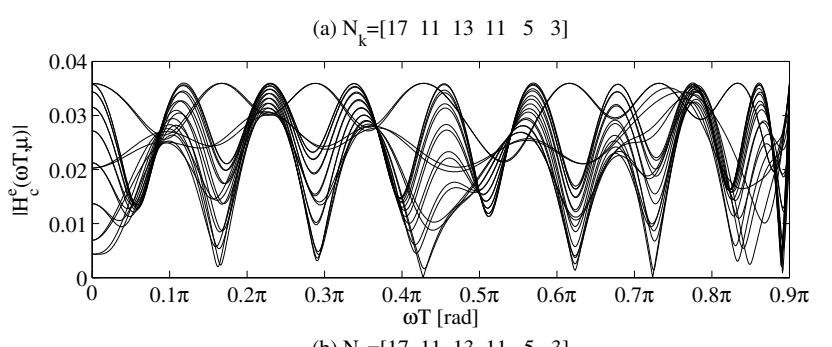

(b) $\mathrm{N}_{\mathrm{k}}=\left[\begin{array}{llllll}17 & 11 & 13 & 11 & 5 & 3\end{array}\right]$

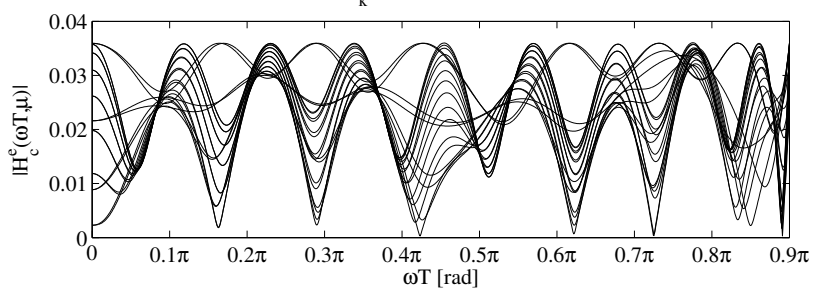

Fig. 7. Complex error for low-delay FD filters with $\Delta=5.5$. (a) Example 6. (b) Example 7.

with a reduced order for these linear-phase FIR filters, the approximation error is not affected. However, the multiplicative complexity is reduced by an average of $30 \%$. The paper also showed that we can have subfilters of different orders so as to further reduce the arithmetic complexity. A framework to systematically obtain these orders is a topic for future work.

\section{REFERENCES}

[1] U. Zölzer, Digital Audio Signal Processing. New York: Wiley, 1997.

[2] W. H. T. (Editor), Software Defined Radio: Enabling Technologies. New York: Wiley, 2002.

[3] S. R. Dooley and A. K. Nandi, "On explicit time delay estimation using the Farrow structure," Signal Processing, vol. 72, pp. 53-57, 1999.

[4] H. Johansson and P. Löwenborg, "Reconstruction of nonuniformly sampled bandlimited signals by means of digital fractional delay filters," IEEE Trans. Signal Processing, vol. 50, no. 11, pp. 2757-2767, Nov. 2002.

[5] J. H. Reed, Software Radio: A Modern Approach to Radio Engineering. Prentice Hall; NJ, 2002.

[6] C. W. Farrow, "A continuously variable digital delay element," in Proc. IEEE Int. Symp. Circuits Syst., vol. 3, Espoo, Finland, June 1988, pp. 2641-2645.

[7] J. Vesma and T. Saramäki, "Design and properties of polynomial-based fractional delay filters," in Proc. IEEE Int. Symp. Circuits Syst., Geneva, Switzerland, 2000.

[8] H. Johansson and P. Löwenborg, "On the design of adjustable fractional delay FIR filters,” IEEE Trans. Circuits Syst. II, vol. 50, no. 4, pp. 164169, Apr. 2003.

[9] J. Yli-Kaakinen and T. Saramäki, "Multiplication-free polynomial-based FIR filters with an adjustable fractional delay," Circuits Syst. Signal Processing, vol. 25, no. 2, pp. 265-294, Apr. 2006.

[10] T. I. Laakso, V. Välimaki, M. Karjalainen, and U. K. Laine, "Splitting the unit delay-tools for fractional delay filter design," IEEE Signal Processing Mag., vol. 13, no. 1, pp. 30-36, Jan. 1996.

[11] C. K. S. Pun, Y. C. Wu, S. C. Chan, and K. L. Ho, "On the design and efficient implementation of the Farrow structure," IEEE Signal Processing Lett., vol. 10, no. 7, pp. 189-192, July 2003.

[12] E. Hermanowicz and H. Johansson, "On designing minimax adjustable wideband fractional delay FIR filters using two-rate approach," in Proc. Eur. Conf. Circuit Theory Design, Cork, Ireland, Aug. 29-Sept. 12005.

[13] S. K. Mitra, Digital Signal Processing: A Computer Based Approach. McGraw-Hill, Feb. 2006. 